\title{
Completing partial latin squares with one nonempty row, column, and symbol
}

\author{
Jaromy Kuhl \\ Department of Mathematics \\ University of West Florida \\ Pensacola, FL, U.S.A. \\ jkuhl@uwf.edu
}

\author{
Michael W. Schroeder \\ Department of Mathematics \\ Marshall University \\ Huntington, WV, U.S.A \\ schroederm@marshall.edu
}

Submitted: Nov 3, 2015; Accepted: Apr 19, 2016; Published: Apr 29, 2016

Mathematics Subject Classifications: 05A05, 05B15

\begin{abstract}
Let $r, c, s \in\{1,2, \ldots, n\}$ and let $P$ be a partial latin square of order $n$ in which each nonempty cell lies in row $r$, column $c$, or contains symbol $s$. We show that if $n \notin\{3,4,5\}$ and row $r$, column $c$, and symbol $s$ can be completed in $P$, then a completion of $P$ exists. As a consequence, this proves a conjecture made by Casselgren and Häggkvist. Furthermore, we show exactly when row $r$, column $c$, and symbol $s$ can be completed.
\end{abstract}

\section{Introduction}

Let $n$ be a positive integer and $S$ a symbol set of cardinality $n$. A partial latin square of order $n$ is an $n \times n$ matrix partially filled with symbols from $S$ such that each symbol occurs at most once in each row and column. Rows and columns are indexed with the set $[n]=\{1,2, \ldots, n\}$ and $S=[n]$, unless otherwise stated. The set of all partial latin squares of order $n$ is denoted $\operatorname{PLS}(n)$.

Let $P \in \operatorname{PLS}(n)$. The symbol located in cell $(i, j)$ of $P$, if such a symbol exists, is denoted $P(i, j)$. We will often write $P$ as a subset of $[n] \times[n] \times S$ in which $(i, j, k) \in P$ if and only if $P(i, j)=k$. If cell $(i, j)$ of $P$ is nonempty for all $i, j \in[n]$, then $P$ is called a latin square and we write $P \in \operatorname{LS}(n)$. We say that $P$ is completable if there is a latin square $L \in \operatorname{LS}(n)$ in which $P(i, j)=L(i, j)$ for each nonempty cell $(i, j)$ of $P$, or alternatively, $P \subseteq L$. 
The problem of completing partial latin squares is notoriously difficult. For an arbitrary $P \in \operatorname{PLS}(n)$, determining whether or not it is completable is NP-complete [3]. However, there are known families of completable partial latin squares. Marshall Hall [6] proved the following.

Theorem 1.1. Let $r$ and $n$ be integers such that $r \leqslant n$. Let $P \in \operatorname{PLS}(n)$ in which the cells of $r$ rows (columns) are filled and the remaining cells empty. Then $P$ can be completed to an element of $\operatorname{LS}(n)$.

Theorem 1.1 essentially says that if $P$ contains exactly $r$ filled rows or $r$ filled columns, then $P$ can be completed. The same can be said of symbols. If $P$ contains exactly $r$ symbols, each occurring $n$ times, then $P$ can be completed. Furthermore, by rearranging the completed $n-r$ rows (columns) from Theorem 1.1, we have the following corollary.

Corollary 1.2. Let $P \in \operatorname{PLS}(n)$ with exactly $r$ filled rows (columns) and one filled column (row). Then $P$ is completable.

Other families of completable partial latin squares come from solutions to the famous Evans Conjecture [4].

Theorem 1.3. All partial latin squares of order $n$ with at most $n-1$ nonempty cells are completable.

Proofs of Theorem 1.3 were given independently by Häggkvist for $n \geqslant 1111$ [5], and by Andersen and Hilton [1] and Smetaniuk [7] for all $n$. The upper bound on the number of nonempty cells is sharp. In [1], the authors determine all incompletable partial latin squares of order $n$ with exactly $n$ nonempty cells. One such square has symbol 1 in the first $k$ diagonal cells and symbols $2,3, \ldots, n-k+1$ in the last $n-k$ cells of column $k+1$. We name this partial latin square $B_{k, n}$.

Let $P \in \operatorname{PLS}(n)$ and $S_{n}$ be the symmetric group acting on $[n]$. For $\theta=(\alpha, \beta, \gamma) \in$ $S_{n} \times S_{n} \times S_{n}$, we use $\theta(P) \in \operatorname{PLS}(n)$ to denote the array in which the rows, columns, and symbols of $P$ are permuted according to $\alpha, \beta$, and $\gamma$ respectively. The mapping $\theta$ is called an isotopism, and $P$ and $\theta(P)$ are said to be isotopic. A conjugate of $P$ is an array in which the coordinates of each triple of $P$ are uniformly permuted. There are six, not necessarily distinct, conjugates of $P$. The main class, or species of $P$ is the set of all partial latin squares that are isotopic to some conjugate of $P$.

The following theorem is Andersen and Hilton's solution to the Evans conjecture [1].

Theorem 1.4. If $P \in \mathrm{PLS}(n)$ with exactly $n$ nonempty cells, then $P$ is completable if and only if $P$ is not a species of $B_{k, n}$ for each $k \in[n-1]$.

More recently, the following family was studied in [2]. For $n>0$, we say that $P \in \operatorname{PLS}(n)$ satisfies the $R C S$ property if there exists $r, c, s \in[n]$ such that for all $(x, y, z) \in P$, either $x=r, y=c$, or $z=s$. Casselgren and Häggkvist conjectured the following in [2]. 
Conjecture 1.5. Let $n>0, P \in \operatorname{PLS}(n)$, and $r, c$, and $s$ defined as above. If $P$ satisfies the RCS property, $(r, c, s) \in P$, and $n \notin\{3,4,5\}$, then $P$ is completable.

Observe that Conjecture 1.5 is trivial for $n=1$ and $n=2$. Casselgren and Häggkvist confirmed Conjecture 1.5 when $n \in\{6,7\}$ and $n=4 k$ for all integers $k \geqslant 2$ [2]. Arrays (a), (b), and (c) in Figure 1 illustrate why the conjecture excludes orders 3, 4, and 5.

\begin{tabular}{|l|l|l|}
\hline 1 & 2 & 3 \\
\hline 2 & 1 & \\
\hline 3 & & 1 \\
\hline
\end{tabular}

(a)

\begin{tabular}{|l|l|l|l|}
\hline 1 & 3 & 4 & 2 \\
\hline 2 & 1 & & \\
\hline 3 & & 1 & \\
\hline 4 & & & 1 \\
\hline
\end{tabular}

(b)

\begin{tabular}{|l|l|l|l|l|}
\hline 1 & 3 & 2 & 4 & 5 \\
\hline 2 & 1 & & & \\
\hline 3 & & 1 & & \\
\hline 4 & & & 1 & \\
\hline 5 & & & & 1 \\
\hline
\end{tabular}

(c)

\begin{tabular}{|l|l|l|l|l|}
\hline 2 & 3 & & 4 & 5 \\
\hline & 1 & & & \\
\hline 3 & & 1 & & \\
\hline 4 & & & 1 & \\
\hline 5 & & & & 1 \\
\hline
\end{tabular}

(d)

Figure 1: Incompletable partial latin squares.

For $n>0$, define $\mathcal{P}_{n} \subseteq \operatorname{PLS}(n)$ as the partial latin squares that satisfy the RCS property and do not contain a species of $B_{k, n}$ for each $k \in[n-1]$; these species are called forbidden configurations. Clearly all completable partial latin squares satisfying the RCS property belong to $\mathcal{P}_{n}$. Array (d) in Figure 1 contains a forbidden configuration and thus does not belong to $\mathcal{P}_{5}$.

In this paper, we prove the following:

Theorem 1.6. Let $n \geqslant 6$. If $P \in \mathcal{P}_{n}$, then $P$ is completable.

In Section 2, we show that for each $P \in \mathcal{P}_{n}$, row $r$, column $c$, and symbol $s$ can be completed. Then, in Section 3, we prove Conjecture 1.5 for $n \geqslant 8$. In Section 4, we show that if $n \geqslant 6, P \in \mathcal{P}_{n}$, and a completion of row $r$, column $c$, and symbol $s$ does not include $(r, c, s)$, then $P$ is completable.

Observe that if $P \in \operatorname{PLS}(n)$ with the RCS property, then each of its species also has the RCS property. Thus, we assume that $r=c=s=1$ throughout this paper.

\section{Necessary Conditions}

Let $P \in \mathcal{P}_{n}$ such that an empty cell occurs in row 1 or column 1 , or symbol 1 appears fewer than $n$ times. We say that $P$ has an $R C S$ completion if row 1 , column 1 , and symbol 1 can be completed. We show that an RCS completion of $P$ exists.

Observation 2.1. If $P(1,1)=1$, then $P$ has an RCS completion by arbitrarily filling row 1 and column 1 , and adding symbol 1 as necessary. 
Lemma 2.2. Let $P \in \mathcal{P}_{n}$ and suppose that cell $(1,1)$ of $P$ is empty. Then $P$ has an $R C S$ completion.

Proof. Let $S$ be the union of symbols appearing in either row 1 or column 1 . Since $P$ contains no forbidden configurations, $S \neq[n]$. If $1 \notin S$, set $P(1,1)=1$ and the result follows from Observation 2.1. Otherwise, let $x \in[n] \backslash S$ and place $x$ in cell $(1,1)$. Without loss of generality, assume that 1 appears in row 1.

Suppose that 1 does not appear in column 1 and cannot be added to column 1 . Suppose that $P$ contains $k$ occurrences of 1 . Without loss of generality, assume they occur in the first $k$ rows. It follows that the latter $n-k$ cells in column 1 must be filled which, when combined with the $k 1$ 's, yield a forbidden configuration. Thus, symbol 1 can be added to column 1. Since 1 appears in column 1 and row 1, symbol 1 can be completed. It follows that row 1 and column 1 can be completed.

Lemma 2.3. Let $P \in \mathcal{P}_{n}$ and suppose that cell $(1,1)$ of $P$ is nonempty. Then $P$ has an RCS completion.

Proof. If $P(1,1)=1$, then the result follows from Observation 2.1, so assume that $P(1,1) \neq 1$. If 1 does not appear in row 1 , then the conjugate of $P$ obtained by swapping symbol and column coordinates has an empty $(1,1)$ cell, and so the result follows from Lemma 2.2. A similar result holds if 1 does not appear in column 1 . If 1 appears in both row 1 and column 1, then $P$ has an RCS completion as outlined at the end of the proof of Lemma 2.2.

\section{A proof for Conjecture $\mathbf{1 . 5}$}

For this section, we consider only elements $P \in \mathcal{P}_{n}$ for which an RCS completion of $P$ includes $(1,1,1)$. Let $F_{n} \subseteq S_{n}$ be the set of permutations which fix 1 , and for each $\pi \in F_{n}$, define $P_{\pi} \in \operatorname{PLS}(n)$ as $\{(a, 1, a),(1, a, \pi(a)),(a, a, 1) \mid a \in[n]\}$. Observe that if $P \in \mathcal{P}_{n}$, then each RCS completion of $P$ is isotopic to $P_{\pi}$ for some $\pi \in F_{n}$.

In what follows we show that for each $\pi \in F_{n}, P_{\pi}$ is completable when $n \geqslant 8$. This, in conjunction with Lemmas 2.2 and 2.3, completes our proof of Conjecture 1.5. We achieve this using semi-invariant permutations.

\subsection{Semi-Invariant Permutations}

Let $\pi \in F_{n}$. Define $\pi$ as semi-invariant over a set $I \subseteq[n]$ if $|I|=\lfloor n / 2\rfloor, 1 \in I$, and $\pi$ is invariant on $I$ (in other words, fixes $I$ setwise). We say that $\pi$ is semi-invariant if such a set $I$ exists. The reduction $\pi(I)$ is the restriction of $\pi$ to $I$. 
Example 3.1. Let $\pi_{1}, \pi_{2} \in F_{8}$ and $\pi_{3}, \pi_{4} \in F_{9}$ be the permutations $\pi_{1}=(1)(2345)(678)$, $\pi_{2}=(1)(2345678), \pi_{3}=(1)(234)(56)(78)(9)$, and $\pi_{4}=(1)(2934)(56)(78)$, given in disjoint cycle notation. Observe that $\pi_{1}$ is semi-invariant over $\{1,6,7,8\}, \pi_{3}$ is semiinvariant over $\{1,2,3,4\},\{1,5,6,9\}$, and $\{1,7,8,9\}$, and both $\pi_{2}$ and $\pi_{4}$ are not semiinvariant. Reductions of $\pi_{1}$ and $\pi_{3}$ are $\pi_{1}(\{1,6,7,8\})=(1)(678), \pi_{3}(\{1,2,3,4\})=$ $(1)(234), \pi_{3}(\{1,5,6,9\})=(1)(56)(9)$, and $\pi_{3}(\{1,7,8,9\})=(1)(78)(9)$.

We use the following observation when a permutation is not semi-invariant.

Observation 3.2. Let $\pi \in F_{n}$ and suppose $\pi$ is not semi-invariant. There exists a transposition $(a b)$ so that $(a b) \pi$ is semi-invariant over a set $I$, with $a \in I$ and $b \notin I$.

Example 3.3. Let $\pi_{2}=(1)(2345678)$ and $\pi_{4}=(1)(2934)(56)(78)$, as given in Example 3.1. Both $\pi_{2}$ and $\pi_{4}$ are not semi-invariant, but $(25) \pi_{2}$ is semi-invariant over $\{1,2,3,4\}$ and $(39) \pi_{4}=\pi_{3}$ is semi-invariant over $\{1,7,8,9\}$.

\subsection{Standard Forms}

Let $\pi \in F_{n}$ be semi-invariant over a set $I$. After appropriate row and column permutations, we may assume that $P_{\pi}$ has the form given in Figure 2 (a). If $\pi$ is not semi-invariant, there exists $I \subset[n]$ and $a, b \in[n]$ so that $(a b) \pi$ is semi-invariant as in Observation 3.2. Let $c, d \in[n]$ such that $a=\pi(c)$ and $b=\pi(d)$. After appropriate row and column permutations, we may assume that $P_{\pi}$ has the form given in Figure 2 (b). We say that these forms are standard forms with respect to $I$. The rows and columns moved to be the first $\lfloor n / 2\rfloor$ rows and columns in Figure 2 (a) and (b) are those indexed by $I$. When we assume standard forms, we keep $I$ and $[n] \backslash I$ as the indices.

Example 3.4. Let $\pi_{3}$ and $\pi_{4}$ be as given in Example 3.1. Recall that $\pi_{3}=(39) \pi_{4}$ is semi-invariant over $\{1,7,8,9\}$. Standard forms for $\pi_{3}$ and $\pi_{4}$ over $\{1,7,8,9\}$ are given in Figure 3 (a) and (b).

The next observation shows the relationship between $P_{\pi}$ and $P_{(a b) \pi}$ when $\pi$ is not semiinvariant and $(a b) \pi$ is semi-invariant. Assume that $P_{\pi}$ is in standard form (see Figure 2 (b)).

Observation 3.5. Let $\pi \in F_{n}$ and $a, b, c, d \in[n] \backslash\{1\}$ with $\pi(c)=a$ and $\pi(d)=b$, then

$$
P_{\pi} \cup\{(c, c, 1),(d, d, 1)\} \backslash\{(d, c, 1),(c, d, 1)\}=P_{(a b) \pi} .
$$

In what follows, if a standard form is needed for $P_{\pi}$, we will state it. 


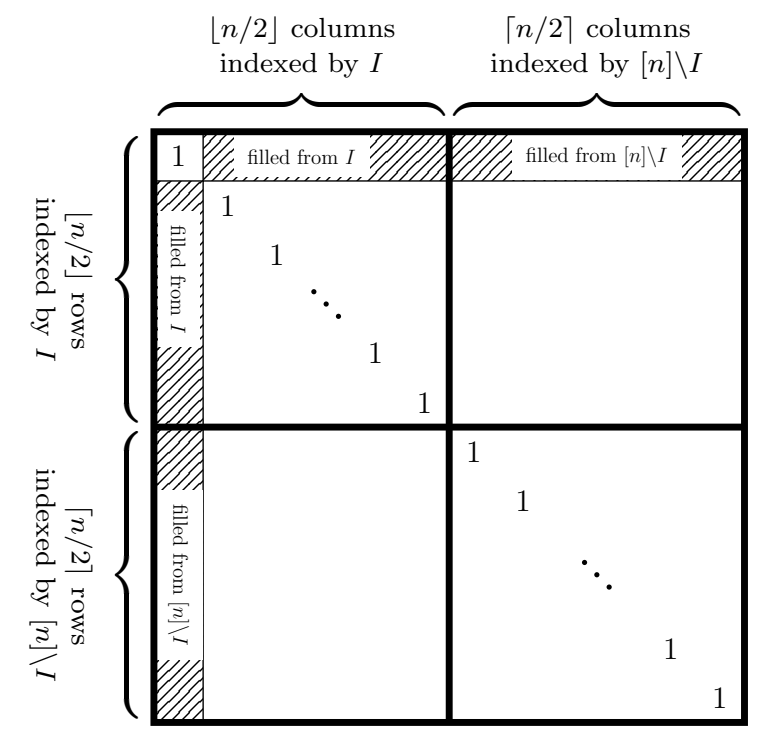

(a)

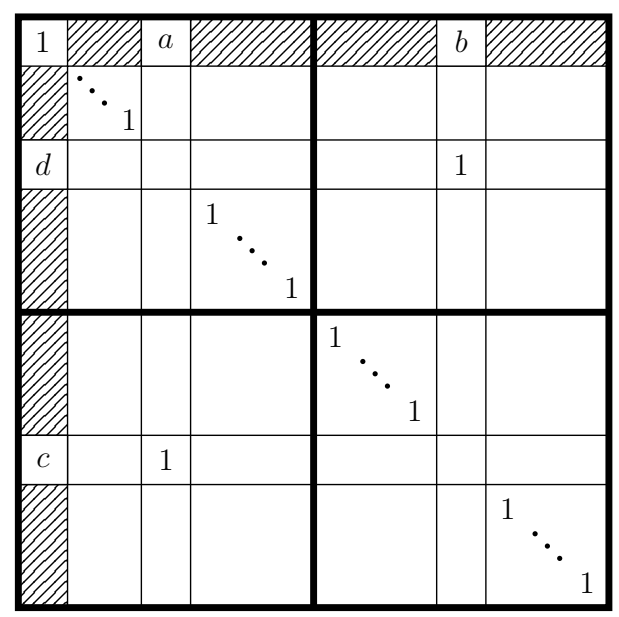

(b)

Figure 2: Standard forms of $P_{\pi}$ with respect to $I$ if $\pi$ is (a) semi-invariant or (b) not semi-invariant.

\subsection{Adding a Partial Transversal}

The following lemma will be used to prove Conjecture 1.5. A partial transversal of length $n-1$ is an element of $\operatorname{PLS}(n)$ consisting of exactly $n-1$ triples, no two of which agree in a row, column, or symbol coordinate.

Lemma 3.6. Let $n \geqslant 6$ and $\pi \in F_{n}$. There is a partial latin square of order $n$ containing $P_{\pi}$ and, disjoint from $P_{\pi}$, a partial transversal of length $n-1$.

Proof. We begin by identifying the cells of the partial transversal to be added to $P_{\pi}$. These cells are $\{(2,3),(3,4), \ldots,(n-1, n)(n, 2)\}$.

Let $G$ be the bipartite graph with vertex parts $\left\{r_{2}, \ldots, r_{n}\right\}$ and $\{2, \ldots, n\}$. We may think of the vertex parts as the rows and symbols of $P_{\pi}$ respectively. Edge $r_{i} j$ is included in $G$ if and only if $j \neq i$ and $j \neq \pi(i+1)$. It follows that the degree of each vertex is at least $(n-1)-2 \geqslant \frac{n}{2}$ since $n \geqslant 6$. Thus, a perfect matching $M=\left\{\left(r_{2}, j_{2}\right),\left(r_{3}, j_{3}\right), \ldots,\left(r_{n}, j_{n}\right)\right\}$ exists in $G$ by Hall's Marriage Theorem.

The partial latin square $P_{\pi} \cup\left\{\left(k, k+1, j_{k}\right) \mid 2 \leqslant k \leqslant n-1\right\} \cup\left\{\left(n, 2, j_{n}\right)\right\}$ contains $P_{\pi}$ and a partial tranversal of length $n-1$ disjoint from $P_{\pi}$.

Example 3.7. Let $\pi=(1)(234)(56) \in F_{6}$. Following the procedure in Lemma 3.6, a partial transversal of length 5 can be added to $P_{\pi}$. See Figure 3 (c). 


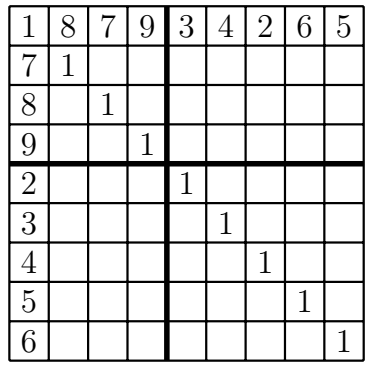

(a)

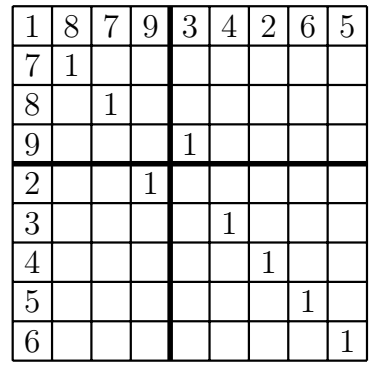

(b)

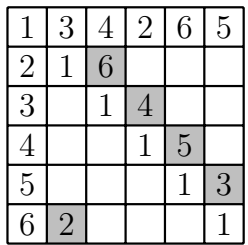

(c)

Figure 3: $\quad$ Standard forms for (a) $P_{\pi_{3}}$ and (b) $P_{\pi_{4}}$ from Example 3.4.

(c) The addition of a partial transversal in $P_{134265}$ from Example 3.7.

\subsection{Main Result}

Lemma 3.8. Let $n \geqslant 8$ and suppose that $\pi \in F_{n}$ is semi-invariant with a reduction $\bar{\pi}$. If $P_{\bar{\pi}}$ is completable, then $P_{\pi}$ is completable.

Proof. Let $\pi \in F_{n}$ over $[n]$ be semi-invariant with respect to $S_{1}$. Without loss of generality, we assume that $P_{\pi}$ is in standard form, and define $S_{2}=[n] \backslash S_{1}$. We begin by partitioning $P_{\pi}$ into four subarrays $P_{k \ell}(k, \ell \in\{1,2\})$ defined as $P_{k \ell}=\left\{(i, j, s) \mid i \in S_{k}, j \in S_{\ell}\right\}$. See Figure 4. Observe that $P_{11}=P_{\bar{\pi}}$, and let $Q_{11}$ be a completion of $P_{\bar{\pi}}$ over $S_{1}$.

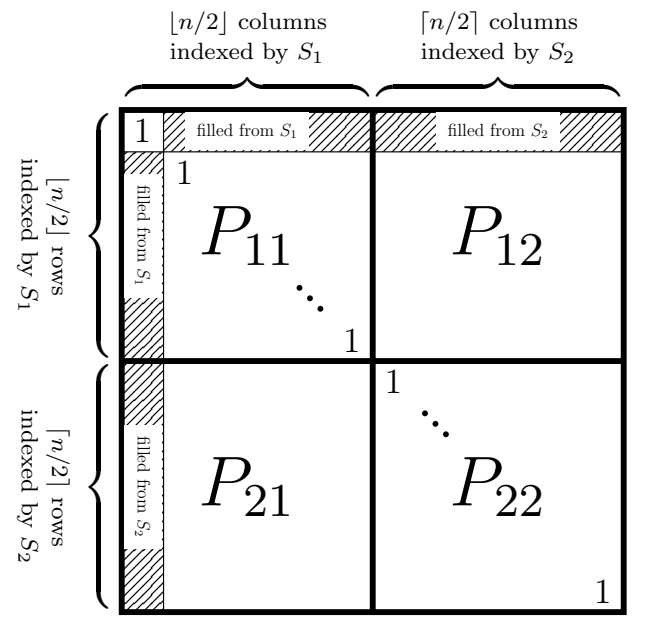

Figure 4: The subarrays of $P_{\pi}$ in Lemma 3.8 .

First, suppose that $n$ is even. Observe that $\left|S_{1}\right|=\left|S_{2}\right|=n / 2$, and so each $P_{k \ell}(k, \ell \in$ $\{1,2\})$ is a partial latin square. Since $P_{12}, P_{21}$, and $P_{22}$ have only a completed row, column, and symbol, respectively, by Theorem 1.1 there exists completions of each over $S_{2}, S_{2}$, and $S_{1}$, respectively. Let $Q_{12}, Q_{21}$, and $Q_{22}$ denote completions of $P_{12}, P_{21}$, and $P_{22}$, respectively. Therefore $Q_{11} \cup Q_{12} \cup Q_{21} \cup Q_{22}$ is a completion of $P_{\pi}$. 
Now suppose that $n$ is odd. Then $\left|S_{1}\right|=(n-1) / 2$ and $\left|S_{2}\right|=(n+1) / 2$. Let $\phi$ be the permutation of order $(n+3) / 2$ induced from $\pi$ over $S_{2} \cup\{1\}$. Let $P_{\phi}$ be the partial latin square of order $(n+3) / 2$ over $\{1\} \cup S_{2}$ with triples:

$\left\{(i, i, 1) \mid i \in\{1\} \cup S_{2}\right\} \cup\left\{(i, 1, s) \mid i \in S_{2},(i, 1, s) \in P_{\pi}\right\} \cup\left\{(1, i, s) \mid i \in S_{2},(1, i, s) \in P_{\pi}\right\}$.

From Lemma 3.6, since $(n+3) / 2 \geqslant 6$, there exists a partial transversal $T$ on symbol set $S_{2}$ of length $(n+1) / 2$ which can be added to $P_{\phi}$.

Let $m$ be a new symbol and define the following partial latin squares of order $(n+1) / 2$ :

$$
\begin{aligned}
& P_{12}^{\prime}=P_{12} \cup\{(m, j, s) \mid(i, j, s) \in T\} \subseteq\left(S_{1} \cup\{m\}\right) \times S_{2} \times S_{2}, \\
& P_{21}^{\prime}=P_{21} \cup\{(i, m, s) \mid(i, j, s) \in T\} \subseteq S_{2} \times\left(S_{1} \cup\{m\}\right) \times S_{2}, \text { and } \\
& P_{22}^{\prime}=P_{22} \cup\{(i, j, m) \mid(i, j, s) \in T\} \subseteq S_{2} \times S_{2} \times\left(S_{1} \cup\{m\}\right) .
\end{aligned}
$$

In other words, $P_{12}^{\prime}, P_{21}^{\prime}$, and $P_{22}^{\prime}$ are each obtained by adding a new filled row to $P_{12}$, a new filled column to $P_{21}$, and by adding $(n+1) / 2$ copies of $m$ to the cells of $P_{22}$. By Theorem 1.1, $P_{12}^{\prime}, P_{21}^{\prime}$, and $P_{22}^{\prime}$ each have completions over $S_{2}, S_{2}$, and $S_{1} \cup\{m\}$ respectively, which we name $Q_{12}, Q_{21}$, and $Q_{22}$.

By removing the row and column indexed by $m$ in $Q_{12}$ and $Q_{21}$, replacing the occurrences of symbol $m$ in $Q_{22}$ with $T$, and combining these with $Q_{11}$, we construct a completion of $P_{\pi}$. Formally,

$Q_{11} \cup\left\{(i, j, k) \in Q_{12} \mid i \neq m\right\} \cup\left\{(i, j, k) \in Q_{21} \mid j \neq m\right\} \cup\left\{(i, j, k) \in Q_{22} \mid k \neq m\right\} \cup T$ is a completion of $P_{\pi}$.

Example 3.9. Let $\pi=(1)(78)(9)(234)(56)$. In Figure 5, we illustrate the arrays needed to complete $P_{\pi}$. Observe that $\pi$ is semi-invariant over $\{1,7,8,9\}$ with reduction $\bar{\pi}=$ (1)(78)(9) and $P_{\bar{\pi}}$ has a completion $Q_{11}$. Completions of $P_{12}^{\prime}, P_{21}^{\prime}$, and $P_{22}^{\prime}$, as outlined in Lemma 3.8, are given in Figure 5 (a). A completion of $P_{\pi}$ is given in Figure 5 (b). The $\phi$ and partial transversal used to complete $P_{\pi}$ are the ones in Figure 3 (c).

In what follows, we consider permutations $\pi \in F_{n}$ which are not semi-invariant. We first find a transposition $(a b)$ so that $(a b) \pi$ is semi-invariant, then find a particular completion of $P_{(a b) \pi}$, and finally perform a slight modification that involves intercalates to produce a completion of $P_{\pi}$.

Definition 3.10. Suppose that $L \in \mathrm{LS}(n)$ and $L$ contains an intercalate $M$ - a subset of $L$ of the form $M=\left\{(i, j, k),\left(i^{\prime}, j, k^{\prime}\right),\left(i, j^{\prime}, k^{\prime}\right),\left(i^{\prime}, j^{\prime}, k\right)\right\}$, for some $i, j, k, i^{\prime}, j^{\prime}, k^{\prime} \in[n]$ where $i \neq i^{\prime}, j \neq j^{\prime}$, and $k \neq k^{\prime}$. The switch of $M$ is $\left\{\left(i, j, k^{\prime}\right),\left(i^{\prime}, j, k\right),\left(i, j^{\prime}, k\right),\left(i^{\prime}, j^{\prime}, k^{\prime}\right)\right\}$, and the switch of $L$ with respect to $M$ is obtained by removing $M$ from $L$ and adding the switch of $M$. Observe that the switch of $L$ with respect to $M$ is an element of $\operatorname{LS}(n)$.

Lemma 3.11. Let $n \geqslant 8$ and suppose that $\pi \in F_{n}$ is not semi-invariant, but $(a b) \pi$ is semi-invariant with a reduction $\bar{\pi}$ for some $a, b \in[n]$. If $P_{\bar{\pi}}$ is completable, then $P_{\pi}$ is completable. 


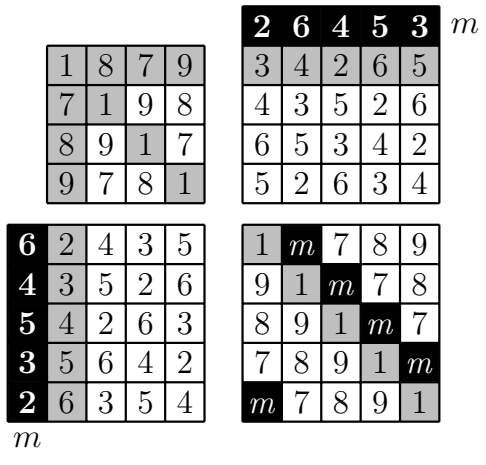

(a)

\begin{tabular}{|l|l|l|l|l|l|l|l|l|}
\hline 1 & 8 & 7 & 9 & 3 & 4 & 2 & 6 & 5 \\
\hline 7 & 1 & 9 & 8 & 4 & 3 & 5 & 2 & 6 \\
\hline 8 & 9 & 1 & 7 & 6 & 5 & 3 & 4 & 2 \\
\hline 9 & 7 & 8 & 1 & 5 & 2 & 6 & 3 & 4 \\
\hline 2 & 4 & 3 & 5 & 1 & $\mathbf{6}$ & 7 & 8 & 9 \\
\hline 3 & 5 & 2 & 6 & 9 & 1 & 4 & 7 & 8 \\
\hline 4 & 2 & 6 & 3 & 8 & 9 & 1 & $\mathbf{5}$ & 7 \\
\hline 5 & 6 & 4 & 2 & 7 & 8 & 9 & 1 & $\mathbf{3}$ \\
\hline 6 & 3 & 5 & 4 & $\mathbf{2}$ & 7 & 8 & 9 & 1 \\
\hline
\end{tabular}

(b)

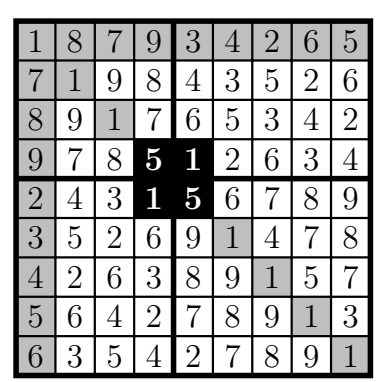

(c)

Figure 5: (a) The latin squares $Q_{k \ell}\left(P_{k \ell}^{\prime}\right.$ is shaded).

(b) A completion of $P_{\pi_{3}}$ from Example 3.9.

(c) A completion of $P_{\pi_{4}}$ from Example 3.12.

Proof. Assume that $P_{\pi}$ is in standard form with respect to $S_{1}$ and let $S_{2}=[n] \backslash S_{1}$, where $a, d \in S_{1}, b, c \in S_{2}$, and $\pi(d)=b$ and $\pi(c)=a$ (see Figure $2(\mathrm{~b})$ ). From Observation $3.5, P_{\pi}=P_{(a b) \pi} \cup\{(d, c, 1),(c, d, 1)\} \backslash\{(d, d, 1),(c, c, 1)\}$. We first complete $P_{(a b) \pi}$ using a completion of $P_{\bar{\pi}}$ (similar to what we did in Lemma 3.8). Define $P_{11}=P_{\bar{\pi}}, P_{12}, P_{21}, P_{22}$ as in Lemma 3.8 .

Suppose that $n$ is even, and observe that $\left|S_{1}\right|=\left|S_{2}\right|=n / 2$. Let $Q_{11}$ be a completion of $P_{11}$ and $Q_{22}$ be a completion of $P_{22}$, both over $S_{1}$. Choose symbol $x \in S_{2}$ such that $x \neq b$ and $x \neq c$. Such a symbol can be chosen since $\left|S_{2}\right| \geqslant 4$.

Define $P_{12}^{\prime}=P_{12} \cup\{(d, c, x)\}$ and $P_{21}^{\prime}=P_{21} \cup\{(c, d, x)\}$. Both $P_{12}^{\prime}$ and $P_{21}^{\prime}$ have completions over $S_{2}$ by Corollary 1.2; $Q_{12}$ and $Q_{21}$ respectively. Thus $Q=Q_{11} \cup Q_{12} \cup Q_{21} \cup Q_{22}$ is a completion of $P_{(a b) \pi}$.

Observe that $Q$ contains the intercalate

$$
M=\{(c, c, 1),(d, d, 1),(c, d, x),(d, c, x)\} .
$$

The switch of $Q$ with respect to $M$ is a completion of $P_{\pi}$.

Now suppose that $n$ is odd with $\left|S_{1}\right|=(n-1) / 2$ and $\left|S_{2}\right|=(n+1) / 2$. Let $Q_{11}$ be a completion of $P_{11}$. Let $\phi$ be the permutation of order $(n+3) / 2$ induced from $(a b) \pi$ over $S_{2} \cup\{1\}$. Let $P_{\phi}$ be the partial latin square of order $(n+3) / 2$ over $\{1\} \cup S_{2}$ with triples:

$$
\begin{gathered}
\left\{(i, i, 1) \mid i \in\{1\} \cup S_{2}\right\} \cup\left\{(i, 1, s) \mid i \in S_{2},(i, 1, s) \in P_{(a b) \pi}\right\} \\
\cup\left\{(1, i, s) \mid i \in S_{2},(1, i, s) \in P_{(a b) \pi}\right\} .
\end{gathered}
$$

From Lemma 3.6, since $(n+3) / 2 \geqslant 6$, there exists a partial transversal $T$ on symbol set $S_{2}$ of length $(n+1) / 2$ which can be added to $P_{\phi}$. 
Choose $x \in S_{2}$ such that $x \neq b, x \neq c, x \neq T(i, c)$ and $x \neq T(c, j)$ for appropriate $i, j \in S_{2}$. Such an $x$ can be chosen since $\left|S_{2}\right| \geqslant 5$.

Let $m$ be a new symbol and define the following partial latin squares of order $m=$ $(n+1) / 2$.

$$
\begin{aligned}
& P_{12}^{\prime}=P_{12} \cup\{(m, j, s) \mid(i, j, s) \in T\} \cup\{(d, c, x)\} \subseteq\left(S_{1} \cup\{m\}\right) \times S_{2} \times S_{2}, \\
& P_{21}^{\prime}=P_{21} \cup\{(i, m, s) \mid(i, j, s) \in T\} \cup\{(c, d, x)\} \subseteq S_{2} \times\left(S_{1} \cup\{m\}\right) \times S_{2}, \text { and } \\
& P_{22}^{\prime}=P_{22} \cup\{(i, j, m) \mid(i, j, s) \in T\} \subseteq S_{2} \times S_{2} \times\left(S_{1} \cup\{m\}\right) .
\end{aligned}
$$

Each of the partial latin squares $P_{12}^{\prime}, P_{21}^{\prime}$, and $P_{22}^{\prime}$ have completions over $S_{2}, S_{2}$, and $S_{1} \cup\{m\}$ respectively; $Q_{12}, Q_{21}$, and $Q_{22}$. The latin square

$Q_{11} \cup\left\{(i, j, k) \in Q_{12} \mid i \neq m\right\} \cup\left\{(i, j, k) \in Q_{21} \mid j \neq m\right\} \cup\left\{(i, j, k) \in Q_{22} \mid k \neq m\right\} \cup T$ is a completion of $P_{(a b) \pi}$ containing the intercalate $M$ in (1). Thus, the switch of $Q$ with respect to $M$ is a completion of $P_{\pi}$.

Example 3.12. Let $\pi_{4}=(1)(2934)(56)(78)$ as given in Example 3.1. A completion of $P_{(39) \pi_{4}}$ can be constructed from Lemma 3.11 with $x=5$ (see Figure 5 (b)). Switching the intercalate in rows and columns 4 and 5 gives a completion of $P_{\pi_{4}}$ (see Figure 5 (c)).

In [2], Casselgren and Häggkvist state that $P_{\pi}$ is completable for each $\pi \in F_{i}$, where $i \in\{6,7,8,9,10\}$. They list all completions when $i \in\{6,7\}$, use their main construction to deduce the conclusion when $i=8$, and state that they use an exhaustive computer search when $i \in\{9,10\}$. To complete our work, it is only necessary to show that $P_{\pi}$ is completable for each $\pi \in F_{11}$. However, our argument can be simply manipulated to prove $P_{\pi}$ is completable for each $\pi \in F_{i}$, where $i \in\{8,9,10\}$ as well. This alleviates the need for a computer search and requires only the methods outlined in this paper, so we give them below as well. For the following arguments, we use the completions given in Figure 6.

\begin{tabular}{|l|l|l|l|}
\hline 1 & 2 & 3 & 4 \\
\hline 2 & 1 & 4 & 3 \\
\hline 3 & 4 & 1 & 2 \\
\hline 4 & 3 & 2 & 1 \\
\hline
\end{tabular}

$P_{1234}$

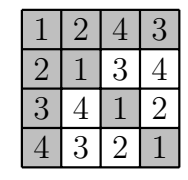

$P_{1243}$

\begin{tabular}{|l|l|l|l|l|}
\hline 1 & 2 & 3 & 4 & 5 \\
\hline 2 & 1 & 4 & 5 & 3 \\
\hline 3 & 5 & 1 & 2 & 4 \\
\hline 4 & 3 & 5 & 1 & 2 \\
\hline 5 & 4 & 2 & 3 & 1 \\
\hline
\end{tabular}

$P_{12345}$

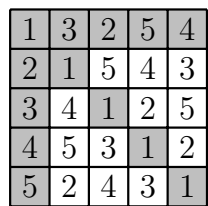

$P_{13254}$

\begin{tabular}{|l|l|l|l|l|}
\hline 1 & 3 & 4 & 2 & 5 \\
\hline 2 & 1 & 3 & 5 & 4 \\
\hline 3 & 5 & 1 & 4 & 2 \\
\hline 4 & 2 & 5 & 1 & 3 \\
\hline 5 & 4 & 2 & 3 & 1 \\
\hline
\end{tabular}

$P_{13425}$

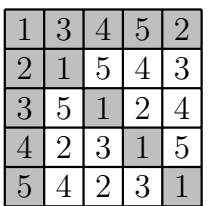

$P_{13452}$

Figure 6: Completions of selected elements of $\mathcal{P}_{4}$ and $\mathcal{P}_{5}$.

Note that the proof of Lemma 3.11 only requires that $(a b) \pi$ be semi-invariant. In the following small cases, it may be that $\pi$ and $(a b) \pi$ are both semi-invariant, however, any reduction of $\pi$ may not be completable, whereas a reduction of $(a b) \pi$ is completable. In what follows, we say a prefix of a permutation $\pi$ is a sequence of the form $\pi(1) \pi(2) \cdots \pi(k)$, or in other words, the first $k$ terms of the single-word representation of $\pi$ for some $k \in[n]$. Before proving our base cases, we make the following observation. 
Observation 3.13. Let $\pi, \pi^{\prime} \in F_{n}$ for some positive $n$. If $P_{\pi}$ is completable and $\pi$ and $\pi^{\prime}$ are conjugates, then $P_{\pi^{\prime}}$ is also completable. Hence we only need to show that $P_{\pi}$ is completable for a permutation $\pi$ from each conjugacy class in $F_{n}$.

Lemma 3.14. If $\pi \in F_{8} \cup F_{9}$, then $P_{\pi}$ is completable.

Proof. By Lemmas 3.8 and 3.11, the completions in Figure 6, and Observation 3.13, it is sufficient to show that for each $\pi \in F_{8} \cup F_{9}$, there is a permutation $\pi^{\prime}$ which is conjugate to $\pi$ such that either

(i) the prefix of $\pi^{\prime}$ is 1234 or 1243 ,

(ii) there exist symbols $a, b$ for which $(a b) \pi^{\prime}$ has a prefix of 1234 or 1243 , or

(iii) a completion of $\pi^{\prime}$ can be found by brute force.

Let $\pi \in F_{i}$ where $i \in\{8,9\}$, and let $\pi_{1}$ be the restriction of $\pi$ to $\{2,3, \ldots, i\}$. We consider the following five cases.

(a) Suppose $\pi_{1}$ consists of at least three 1-cycles. Then $\pi$ has a conjugate whose prefix is 1234 , and hence $P_{\pi}$ is completable.

(b) Suppose that $\pi_{1}$ consists of at least one 1-cycle and at least one 2-cycle. Then $\pi$ has a conjugate whose prefix is 1243 , and hence $P_{\pi}$ is completable.

(c) Suppose that $\pi_{1}$ has no 1-cycle and consists of at least one 2-cycle. Then $\pi$ has a conjugate $\pi^{\prime}$ whose prefix is 1543 . Then $(25) \pi^{\prime}$ has a prefix of 1243 , and hence $P_{\pi^{\prime}}$ and $P_{\pi}$ are completable.

(d) Suppose that $\pi_{1}$ consists of no 2-cycles and either one or two 1-cycles. Then $\pi_{1}$ contains a cycle of length at least 3 . Then $\pi$ has a conjugate $\pi^{\prime}$ whose prefix is 1245 . Then (35) $\pi^{\prime}$ has a prefix of 1243 , and hence $P_{\pi^{\prime}}$ and $P_{\pi}$ are completable.

(e) Suppose that $\pi_{1}$ does not meet any of the above conditions. Then $\pi$ is a conjugate of (1)(2345678), (1)(234)(6789), (1)(23456789), (1)(2345)(6789), or (1)(234)(56789). These have their completions given in Figure 7.
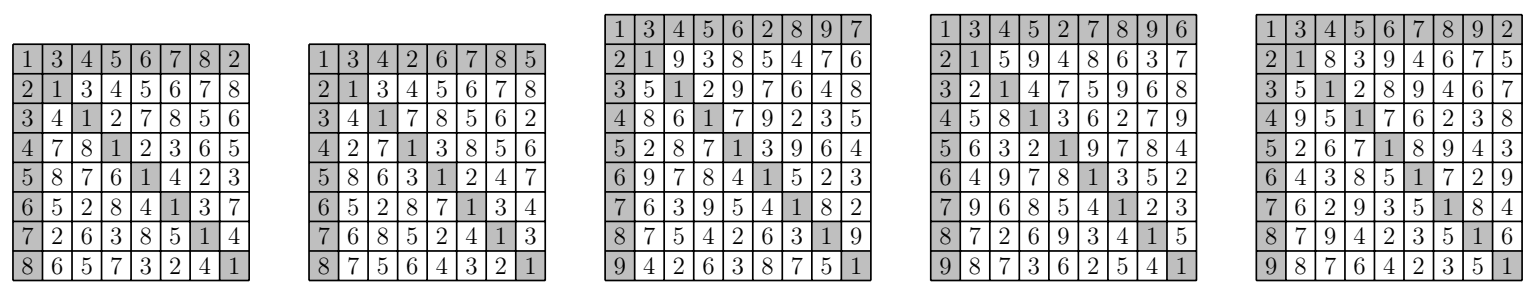

Figure 7: Completions of $P_{13456782}, P_{13426785}, P_{13452897}, P_{134527896}$, and $P_{134567892}$.

Hence $P_{\pi}$ is completable for every $\pi \in F_{8} \cup F_{9}$.

Lemma 3.15. If $\pi \in F_{10} \cup F_{11}$, then $P_{\pi}$ is completable.

Proof. With an argument similar to the proof in Lemma 3.14, we can apply Lemmas 3.8 and 3.11, along with Observation 3.13 the completions in Figure 6. We need only show 
that in each $\pi \in F_{10} \cup F_{11}$, there is a permutation $\pi^{\prime}$ which is conjugate to $\pi$ for which either

(i) the prefix of $\pi^{\prime}$ is $12345,13254,13425$, or 13452 , or

(ii) there exist symbols $a, b$ for which $(a b) \pi^{\prime}$ has a prefix of $12345,13254,13425$, or 13452.

Let $\pi \in F_{i}$ where $i \in\{10,11\}$, and let $\pi_{1}$ be the restriction of $\pi$ to $\{2,3, \ldots, i\}$. We consider the following cases.

(a) Suppose that $\pi_{1}$ has a cycle of length at least 5. Then $\pi$ has a conjugate $\pi^{\prime}$ whose prefix is 13456 . Then $(26) \pi$ has the prefix 13452, and hence $P_{\pi^{\prime}}$ and $P_{\pi}$ are completable.

(b) Suppose that $\pi_{1}$ has a cycle of length 4 and none larger. Then $\pi$ has a conjugate whose prefix is 13452 , and hence $P_{\pi}$ is completable.

(c) Suppose that $\pi_{1}$ has a cycle of length 3 and none larger. If $\pi_{1}$ also has a 1-cycle, then $\pi$ has a conjugate whose prefix is 13425 , and hence $P_{\pi}$ is completable. Otherwise, $\pi$ has a conjugate $\pi^{\prime}$ whose prefix is 13426 . Then (56) $\pi^{\prime}$ has a prefix of 13425 , and hence $P_{\pi^{\prime}}$ and $P_{\pi}$ are completable.

(d) Suppose that $\pi_{1}$ has at least two 2-cycles and none larger. Then $\pi$ has a conjugate whose prefix is 13254 , and hence $P_{\pi}$ is completable.

(e) If $\pi_{1}$ does not satisfy any of the previous conditions, then $\pi_{1}$ has at least four 1-cycles. So $\pi$ has a conjugate with a prefix of 12345 , and hence $P_{\pi}$ is completable.

Therefore $P_{\pi}$ is completable for every $\pi \in F_{10} \cup F_{11}$.

Thus, we have proved the following theorem, confirming Conjecture 1.5.

Theorem 3.16. Let $P \in \mathcal{P}_{n}$ where $(r, c, s) \in P$. If $n \notin\{3,4,5\}$, then $P$ is completable.

\section{The cases for which $(r, c, s) \notin P$}

Let $A \in \operatorname{LS}(n)$. The back diagonal of an $n \times n$ array is the set of cells $\{(i, n-i+1) \mid i \in[n]\}$. We build a partial latin square $T(A)$ of order $n+1$ by setting

- $T(A)(i, j)=n+1$ for each $(i, j)$ on the back diagonal,

- $T(A)(i, j)=A(i, j)$ for each $(i, j)$ above the back diagonal, and

- cell $(i, j)$ of $T(A)$ is empty for each $(i, j)$ below the back diagonal.

Smetaniuk's proof of Theorem 1.3 [7] uses the following completion result.

Theorem 4.1. Let $A \in \operatorname{LS}(n)$. Then $T(A) \in \operatorname{PLS}(n+1)$ is completable.

We can now prove Theorem 1.6. 
Proof of Theorem 1.6. By Lemmas 2.2 and 2.3, we may assume that the first row and column of $P$ are filled and that symbol 1 appears $n$ times. If $P(1,1)=1$, then the result follows from Theorem 3.16. So assume $P(1,1) \neq 1$.

Without loss of generality, assume that $(1, n, 1),(n, 1,1) \in P$ and each symbol 1 occurs on the back diagonal of $P$. Let $P^{\prime} \in \operatorname{PLS}(n)$ be the array formed from $P$ by removing each occurrence of symbol 1. Define $Q \in \operatorname{PLS}(n-1)$ over $[n] \backslash\{1\}$ such that $Q(i, j)=P^{\prime}(i, j)$ for all $i, j \in[n-1]$. By Corollary 1.2, $Q$ can be completed to a latin square $L \in \operatorname{LS}(n-1)$ over $[n] \backslash\{1\}$. By Theorem 4.1, $T(L)$ has a completion, and thus $P$ is completable.

\section{References}

[1] L. D. Andersen and A. J. W. Hilton. Thank Evans! Proc. London Math. Soc. (3), 47(3):507-522, 1983.

[2] Carl Johan Casselgren and Roland Häggkvist. Completing partial latin squares with one filled row, column and symbol. Discrete Math., 313(9):1011-1017, 2013.

[3] Charles J. Colbourn. The complexity of completing partial Latin squares. Discrete Appl. Math., 8(1):25-30, 1984.

[4] T. Evans. Embedding incomplete latin squares. Amer. Math. Monthly, 67:958-961, 1960.

[5] R. Häggkvist. A solution of the Evans conjecture for Latin squares of large size. Combinatorics (Proc. Fifth Hungarian Colloq., Keszthely, 1976), Vol. I, 18:495-513, 1978.

[6] Marshall Hall. An existence theorem for Latin squares. Bull. Amer. Math. Soc., 51:387-388, 1945.

[7] Bohdan Smetaniuk. A new construction on Latin squares. I. A proof of the Evans conjecture. Ars Combin., 11:155-172, 1981. 